\title{
Age-Adjusted Risk Factors Associated with Mortality and Mechanical Ventilation Utilization Amongst COVID-19 Hospitalizations-a Systematic Review and Meta-Analysis
}

\author{
Urvish Patel ${ }^{1}$ - Preeti Malik ${ }^{1}$ (D) - Muhammad Shariq Usman ${ }^{2}$ - Deep Mehta ${ }^{3} \cdot$ Ashish Sharma $^{4} \cdot$ Faizan Ahmad Malik $^{5}$. \\ Nashmia Khan ${ }^{1} \cdot$ Tariq Jamal Siddiqi $^{2} \cdot$ Jawad Ahmed $^{2} \cdot$ Achint Patel $^{1} \cdot$ Henry Sacks $^{6}$
}

Accepted: 20 August 2020 / Published online: 29 August 2020

(C) Springer Nature Switzerland AG 2020

\begin{abstract}
The increasing COVID-19 cases in the USA have led to overburdening of healthcare in regard to invasive mechanical ventilation (IMV) utilization as well as mortality. We aim to identify risk factors associated with poor outcomes (IMV and mortality) of COVID-19 hospitalized patients. A meta-analysis of observational studies with epidemiological characteristics of COVID-19 in PubMed, Web of Science, Scopus, and medRxiv from December 1, 2019 to May 31, 2020 following MOOSE guidelines was conducted. Twenty-nine full-text studies detailing epidemiological characteristics, symptoms, comorbidities, complications, and outcomes were included. Meta-regression was performed to evaluate effects of comorbidities, and complications on outcomes using a random-effects model. The pooled correlation coefficient $(r), 95 \% \mathrm{CI}$, and OR were calculated. Of 29 studies $(12,258$ confirmed cases), 17 reported IMV and 21 reported deaths. The pooled prevalence of IMV was $23.3 \%$ (95\% CI: 17.1-30.9\%), and mortality was $13 \%(9.3-18 \%)$. The age-adjusted meta-regression models showed significant association of mortality with male ( $r: 0.14$; OR: $1.15 ; 95 \%$ CI: $\left.1.07-1.23 ; I^{2}: 95.2 \%\right)$, comorbidities including pre-existing cerebrovascular disease $(r: 0.35$; $\left.1.42(1.14-1.77) ; I^{2}: 96.1 \%\right)$, and chronic liver disease $\left(r: 0.08 ; 1.08(1.01-1.17) ; I^{2}: 96.23 \%\right)$, complications like septic shock $(r$ : $\left.0.099 ; 1.10(1.02-1.2) ; I^{2}: 78.12 \%\right)$ and ARDS $\left(r: 0.04 ; 1.04(1.02-1.06) ; I^{2}: 90.3 \%\right)$, ICU admissions $(r: 0.03 ; 1.03(1.03-1.05)$; $\left.I^{2}: 95.21 \%\right)$, and IMV utilization $\left(r: 0.05 ; 1.05(1.03-1.07) ; I^{2}: 89.80 \%\right)$. Similarly, male $\left(r: 0.08 ; 1.08(1.02-1.15) ; I^{2}: 95 \%\right)$, comorbidities like pre-existing cerebrovascular disease $\left(r: 0.29 ; 1.34(1.09-1.63) ; I^{2}: 93.4 \%\right)$, and cardiovascular disease $(r: 0.28$; $\left.1.32(1.1-1.58) ; I^{2}: 89.7 \%\right)$ had higher odds of IMV utilization. COVID-19 patients with comorbidities including cardiovascular disease, cerebrovascular disease, and chronic liver disease had poor outcomes. Diabetes and hypertension had higher prevalence
\end{abstract}

This article is part of the Topical Collection on Covid-19

Electronic supplementary material The online version of this article (https://doi.org/10.1007/s42399-020-00476-w) contains supplementary material, which is available to authorized users.

Preeti Malik

pmalik.ma@gmail.com

Urvish Patel

dr.urvish.patel@gmail.com

Muhammad Shariq Usman

shariqusman@outlook.com

Deep Mehta

dsmehta94@gmail.com

Ashish Sharma

Ashishsharma1412@gmail.com

Faizan Ahmad Malik

dr.faizanahmadmalik@gmail.com

\author{
Nashmia Khan \\ khan_nashmia@outlook.com \\ Tariq Jamal Siddiqi \\ tariq.jamal.siddiqi@gmail.com \\ Jawad Ahmed \\ jawadahmedd13@gmail.com \\ Achint Patel \\ drachintpatel@gmail.com \\ Henry Sacks \\ Henry.Sacks@mountsinai.org
}

Extended author information available on the last page of the article 
but no association with mortality and IMV. Our study results will be helpful in right allocation of resources towards patients who need them the most.

Keywords COVID-19 $\cdot$ Coronavirus disease $\cdot$ SARS-CoV-2 $\cdot 2019-n C o V \cdot$ Severe acute respiratory syndrome $\cdot$ Mortality Mechanical ventilation $\cdot$ COVID-related complications $\cdot$ COVID risk factors

\section{Introduction}

The first confirmed case of coronavirus disease 2019 (COVID-19) in the USA was reported on 20 January 2020 [1]. The USA now has more confirmed cases than any other country in the world. The number of cases exceeds 1.2 million with a death toll crossing 70,000 [2]. COVID-19 disease affects mainly the respiratory system [3] but there are studies showing the involvement of other systems as well $[4,5]$. Studies have shown that a large number of admitted patients required mechanical ventilation $[3,6,7]$. The common point that these studies show is that the majority of these patients had some associated comorbid condition. The prevalence of diabetes is $10.5 \%$ [8] and hypertension is $29 \%$ [9] in the USA indicating how widespread some of these conditions are. Some other studies revealed that certain risk factors like preexisting cardiovascular, cerebrovascular diseases, age $\geq 65$, $\mathrm{CD} 3+\mathrm{CD} 8+\mathrm{T}$ cells $\leq 75$ cell $/ \mu \mathrm{L}$, and cardiac troponin $I \geq$ $0.05 \mathrm{ng} / \mathrm{mL}$ and d-dimer $>1 \mu \mathrm{g} / \mathrm{mL}$ are associated with increased in-hospital mortality [5, 10-12]. Predicting the risk factors associated with the need for IMV and poor prognosis are thus of utmost importance given the overwhelming number of admissions of critical patients to the hospitals.

Studying the correlation of various factors like demographics, comorbidities, and complications in COVID-19 patients with IMV utilization can help to redirect the limited resources towards patients who require them the most. The other aim of the paper is to identify predictors of mortality adjusted by age based on the same parameters. The predictors of mortality will also help clinicians in early identification of such patients in the course of admission which can save lives and decrease mortality due to COVID-19. The objective of this study was to evaluate the risk factors including comorbidities, and complications associated with the poor outcomes amongst COVID-19 patients.

\section{Method}

\section{Endpoints}

Primary aim of this study was to evaluate the risk factors (ageadjusted) associated with poor outcomes (IMV and mortality) amongst patients with confirmed COVID-19 infection. Secondary outcome of the study was to evaluate demographic and clinical characteristics, comorbidities, and complications of COVID-19 patients. We have not considered recovery and ICU admission as outcomes due to variability in the definitions of recovery and utilization of IMV outside ICU.

\section{Search Strategy and Selection Criteria}

A systematic review was performed using MOOSE guidelines [13]. We searched PubMed, Web of Science, Scopus, and medRxiv for observational studies that described characteristics of COVID-19 from December 1, 2019 to May 31, 2020 following keyword/MESH terms: ((COVID-19[Title/ Abstract]) OR coronavirus[Title/Abstract]) OR SARS-CoV2[Title/Abstract] OR 2019-nCoV[Title/Abstract]. All studies describing epidemiology of COVID-19 were included. Literature other than observational studies, non-English literature, non-full text, and animal studies were excluded. Flow diagram of literature search and study selection process is described in eSupplemental file (1).

\section{Study Selection}

Abstracts were reviewed, and articles were retrieved and reviewed for availability of data on epidemiology of COVID-19. Studies mentioned details on IMV and mortality had been selected for quantitative analysis. UP and PM independently screened all identified studies and assessed full texts to decide eligibility. Any disagreement was resolved through discussion with other reviewers (SU and DM).

\section{Data Collection}

From the included studies, data relating to patient characteristics like age and sex, symptoms like headache, fever, cough, diarrhea, dyspnea hemoptysis, myalgia/fatigue, nausea/ vomiting, sore throat, nasal congestion/rhinorrhea, and sputum production, comorbidities and risk factors like smoker, diabetes, hypertension, malignancy, pulmonary disease, chronic liver disease, cerebrovascular disease, and cardiovascular disease, complications like pneumonia, acute respiratory distress syndrome, septic shock, secondary infections, and cardiac complications, details on discharged/recovery and ICU admission, and outcomes like mortality and needs for IMV were collected using prespecified data collection forms by two authors (UP and PM) with a common consensus of 
authors (SU and TJ) upon disagreement. We have presented the study characteristics like publication year, country of origin, and sample size. Data on the following outcomes which were IMV utilization and mortality were extracted.

\section{Assessment of Risk of Bias}

The Newcastle-Ottawa Quality Assessment Scale [14] was used to evaluate the quality of the included studies and the risk of bias.

\section{Statistical Analysis}

We used all studies containing details on epidemiological characteristics in order to calculate pooled prevalence, $95 \%$ confidence interval (CI), and weights of demographic features, symptoms, comorbidities, risk factors, and complications rate amongst COVID-19 patients precisely. Metaregression was performed to evaluate the effects of comorbidities, risk factors, and complications on outcomes of COVID19 patients. We used comprehensive meta-analysis software to estimate correlation coefficient $(r)$ and $95 \%$ confidence interval $(95 \% \mathrm{CI})$ and odds ratios (OR) (e $\mathrm{e}^{\wedge}$ coefficient) with corresponding $95 \% \mathrm{CI}$ were pooled using a random-effects model. The proportion of total between-study variance explained by the model identified using analogous index $\left(R^{2}\right)$ and statistical heterogeneity across studies was reported using the $I^{2}$ statistics. The $I^{2}$ statistic of $>75 \%$ was considered significant heterogeneity. $p<0.05$ was considered significant. Age-adjusted and unadjusted meta-regression were performed. Sensitivity analysis was also performed using the "leave-one-out method" to probe sources of heterogeneity.

\section{Results}

As of May 31, 2020, we included 29 observational studies (eSupplemental file (2)) with 12,258 confirmed cases of COVID-19 patients detailing epidemiological characteristics, symptoms, comorbidities or risk factors, complications, and outcomes including mortality and IMV. Of those 29 studies, 17 studies have reported IMV utilization and 21 studies have reported deaths. The pooled prevalence of IMV was $23.3 \%$ (95\% CI: $17.1-30.9 \% ; p<0.001 ; 1789 / 8804$ patients), and mortality was $13 \%$ (95\% CI: $9.3-18 \% ; p<0.001$; 1267/11252 patients) (Table 1).

In our pooled cohort of confirmed cases of COVID-19, pooled prevalence of male was $57.3 \%$ (95\% CI: $55.1-$ $59.4 \% ; p<0.001 ; 7198 / 12247$ patients). The most common clinical symptoms of COVID-19 patients were fever with pooled prevalence of $85.6 \%$ (95\% CI: 73.6-92.7\%; $p<0.001 ; 5172 / 9163$ ) followed by cough $64.7 \%$ (95\% CI: $57.4-71.4 \% ; p<0.001 ; 2464 / 3863)$, myalgia or fatigue
43.3\% (95\% CI: $35.8-51.2 \%$; $p<0.096 ; 1848 / 3813)$, sputum production or expectoration $33.4 \%$ (95\% CI: $29.1-38.1 \%$; $p<0.001$; 968/2846), and dyspnea $32 \%$ (95\% CI: 23.9 $41.3 \%$; $p<0.001 ; 1259 / 3629)$. Other clinical symptoms included sore throat with pooled prevalence of $17.3 \%$ (95\% CI: $9.1-30.3 \% ; p<0.001 ; 192 / 1344)$, headache $10.7 \%$ (95\% CI: $7.9-14.3 \% ; p<0.001 ; 306 / 2738)$, diarrhea $9.4 \%$ (95\% CI: $6.2-14.1 \% ; p<0.001 ; 400 / 3428)$, nausea or vomiting $7 \%$ (95\% CI: $4.4-10.8 \% ; p<0.001 ; 265 / 3258)$, nasal congestion 7.5\% (95\% CI: $3.1-17.4 \% ; p<0.001 ; 50 / 1082)$, and hemoptysis $2 \%$ (95\% CI: $1.1-3.9 \% ; p<0.001 ; 29 / 1804)$.

Most common coexisting comorbidities were hypertension with pooled prevalence of $28.2 \%$ (95\% CI: $22.1-35.1 \%$; $p<0.001$; 4858/11626), diabetes $15.4 \%$ (95\% CI: $12-19.4 \%$; $p<0.001 ; 2897 / 11680)$, cardiovascular diseases $12.2 \%(95 \%$ CI: $8.9-16.6 \% ; p<0.001 ; 204 / 11664)$, and smoking $8.9 \%$ (95\% CI: $4.2-17.9 \% ; p<0.001 ; 3003 / 8410)$. Most common complications of COVID-19 infection were pneumonia (68.1\%; 95\% CI: $38.8-78.8 \%$; $p=0.221 ; 1518 / 2113$ ), acute respiratory distress syndrome $(29.9 \%$; $95 \%$ CI: $18.5-44.7 \%$; $p=0.009 ; 470 / 2518)$, cardiac complications $(22.3 \% ; 95 \% \mathrm{CI}$ : $12.8-36.1 \% ; p<0.001 ; 357 / 1246)$, and secondary infection (13.8\%; 95\% CI: 5.8-29.3\%; $p<0.001 ; 218 / 1187$ ) (Table 2).

\section{Meta-Regression}

Meta-regression random-effects models quantified the study level impact of comorbidities, risk factors, and complications in COVID-19 patients on IMV utilization, and mortality. Amongst COVID-19 patients, the age-adjusted meta-regression models showed strong association of mortality with male $(r$ : 0.14; OR: 1.15 ; 95\% CI: $1.07-1.23 ; p=0.0001 ; I^{2}: 95.2 \%$ ), comorbidities including pre-existing cerebrovascular disease $\left(r: 0.35\right.$; OR: $1.42 ; 95 \%$ CI: $1.14-1.77 ; p=0.0018 ; I^{2}$ : $96.1 \%)$, and chronic liver disease ( $r: 0.08$; OR: $1.08 ; 95 \% \mathrm{CI}$ : $\left.1.01-1.17 ; p=0.0259 ; I^{2}: 96.23 \%\right)$, complications like septic shock ( $r$ : 0.099; OR: $1.10 ; 95 \%$ CI: $1.02-1.2 ; p=0.0149 ; I^{2}$ : $78.12 \%)$, and acute respiratory distress syndrome (ARDS) $(r$ : 0.04; OR: $1.04 ; 95 \%$ CI: $1.02-1.06 ; p=0.0005 ; I^{2}: 90.3 \%$ ). Mortality odds were higher amongst patients in intensive care unit patients $(r: 0.03$; OR: $1.03 ; 95 \%$ CI: $1.03-1.05 ; p=0.0001$; $\left.I^{2}: 95.21 \%\right)$ and utilized IMV $(r: 0.05$; OR: 1.05 ; $95 \%$ CI: $1.03-$ $\left.1.07 ; p<0.0001 ; I^{2}: 89.80 \%\right)$. Similarly, in age-adjusted metaregression analysis, male $(r: 0.08$; OR: 1.08 ; 95\% CI: 1.02 $1.15 ; p=0.0140 ; I^{2}: 95 \%$ ), comorbidities like pre-existing cerebrovascular disease $(r: 0.29$; OR: 1.34 ; 95\% CI: 1.09-1.63; $\left.p=0.0038 ; I^{2}: 93.4 \%\right)$, cardiovascular disease $(r: 0.28$; OR: 1.32; 95\% CI: $1.1-1.58 ; p=0.0028 ; I^{2}: 89.7 \%$ ), chronic liver disease ( $r: 0.08$; OR: $1.08 ; 95 \%$ CI: $1.03-1.17 ; p=0.0033 ; I^{2}$ : $94.4 \%$ ), and acute respiratory distress syndrome (correlation coefficient: 0.04; OR: 1.04; 95\% CI: $1.03-1.06 ; p=0.0000$; $I^{2}: 77.34 \%$ ) had higher odds of IMV utilization amongst COVID-19 patients. Pre-existing diabetes mellitus ( $r: 0.02$; 
Table 1 Study characteristics describing details on COVID-19

\begin{tabular}{|c|c|c|c|c|}
\hline Study & Country & $\begin{array}{l}\text { Sample size } \\
\text { total study } \\
(n)=29\end{array}$ & $\begin{array}{l}\text { Mortality (events; event rate }(\%)(95 \% \\
\left.\text { CI)*; weight }(\%)^{\#}\right) \text { total study }(n)=21\end{array}$ & $\begin{array}{l}\text { Mechanical ventilation (events; event rate }(\%) \\
\left.(95 \% \mathrm{CI})^{*} \text {; weight }(\%)^{\#}\right) \text { total study }(n)=17\end{array}$ \\
\hline Huang et al., Jan 2020 & China & 41 & $6 ; 14.6(6.7-29) ; 4.9$ & $4 ; 9.8(3.7-23.3) ; 4.99$ \\
\hline Guan et al., Feb 2020 & China & 1099 & $15 ; 1.4(0.8-2.3) ; 5.87$ & $67 ; 6.1(4.8-7.7) ; 7.54$ \\
\hline Zhao et al., Mar 2020 & China & 19 & NA & $0 ; 2.5(0.2-29.8) ; 1.51$ \\
\hline Young et al., Mar 2020 & Singapore & 18 & NA & $1 ; 5.6(0.8-30.7) ; 2.48$ \\
\hline Wang et al., Feb 2020 & China & 138 & $6 ; 4.3(2-9.3) ; 5.04$ & $17 ; 12.3(7.8-18.9) ; 6.85$ \\
\hline Ng et al., Mar 2020 & Singapore & 100 & $0 ; 0.5(0-7.4) ; 1.47$ & NA \\
\hline Spiteri et al., Mar 2020 & Europe & 38 & $1 ; 2.6(0.4-16.5) ; 2.36$ & $1 ; 2.6(0.4-16.5) ; 2.53$ \\
\hline $\begin{array}{l}\text { COVID-19 National Incident } \\
\text { Room Surveillance Team, Mar } \\
2020\end{array}$ & Australia & 71 & $2 ; 2.8(0.7-10.6) ; 3.47$ & NA \\
\hline Xu et al., Feb 2020 & China & 62 & $0 ; 0.8(0-11.5) ; 1.46$ & $1 ; 1.6(0.2-10.6) ; 2.55$ \\
\hline Bajema et al., Feb 2020 & USA & 11 & $1 ; 9.1(1.3-43.9)^{\mathrm{a}} ; 2.26$ & NA \\
\hline Chen et al., Jan 2020 & China & 99 & $11 ; 11.1(6.3-19) ; 5.57$ & NA \\
\hline Yang et al., Feb 2020 & China & 52 & $32 ; 61.5(47.8-73.7)^{\mathrm{b}} ; 5.75$ & $37 ; 71.2(57.5-81.8) ; 6.54$ \\
\hline Wang et al., Mar 2020 & China & 69 & $5 ; 7.2(3-16.3) ; 4.78$ & NA \\
\hline Mo et al., Mar 2020 & China & 155 & NA & $36 ; 23.2(17.2-30.5) ; 7.25$ \\
\hline Arentz et al., Mar 2020 & USA & 21 & $11 ; 52.4(31.8-72.1)^{\mathrm{c}} ; 4.93$ & $15 ; 71.4(49.2-86.6) ; 5.29$ \\
\hline Wu et al., Mar 2020 & China & 201 & $44 ; 21.9(16.7-28.1) ; 6.24$ & $67 ; 33.3(27.2-40.1) ; 7.44$ \\
\hline Zhou et al., Mar 2020 & China & 191 & $54 ; 28.3(22.3-35.1) ; 6.28$ & $58 ; 30.4(24.3-37.3) ; 7.41$ \\
\hline Wang et al., Mar 2020 & China & 339 & $65 ; 19.2(15.3-23.7) ; 6.35$ & $80 ; 23.6$ (19.4-28.4); 7.53 \\
\hline Guo et al., Mar 2020 & China & 187 & $43 ; 23$ (17.5-29.6); 6.23 & $45 ; 24.1(18.5-30.7) ; 7.35$ \\
\hline Richardson et al., Apr 2020 & USA & 5700 & $553 ; 9.7(9-10.5) ; 6.53$ & $1151 ; 20.2(19.2-21.3) ; 7.76$ \\
\hline Goyal et al., Apr 2020 & USA & 393 & $40 ; 10.2$ (7.6-13.6); 6.26 & $130 ; 33.1(28.6-37.9) ; 7.6$ \\
\hline Ruan et al., Mar 2020 & China & 150 & $68 ; 45.3(37.6-53.4)^{\mathrm{d}} ; 6.27$ & $79 ; 52.7$ (44.7-60.5); 7.38 \\
\hline Qian et al., Mar 2020 & China & 91 & $0 ; 0.5(0-8.1) ; 1.47$ & NA \\
\hline Paranjpe et al., Apr 2020 & USA & 2199 & $310 ; 14.1(12.7-15.6) ; 6.51$ & NA \\
\hline Lauer et al., Mar 2020 & China & 181 & NA & NA \\
\hline Chang et al., Feb 2020 & China & 13 & NA & NA \\
\hline Kim et al., Feb 2020 & $\begin{array}{l}\text { South } \\
\text { Korea }\end{array}$ & 28 & NA & NA \\
\hline Qin et al., Mar 2020 & China & 452 & NA & NA \\
\hline Zhang et al., Feb 2020 & China & 140 & NA & NA \\
\hline Total & & 12,258 & $1267 ; 13(9.3-18) ; 100$ & $1789 ; 23.3$ (17.1-30.9); 100 \\
\hline
\end{tabular}

Total number $(n=)$ of patients included for COVID-19 epidemiology evaluation 12,258, mortality prevalence 11,252, and for mechanical ventilation utilization 8804

* Statistically significant at $p<0.001$ except (a) $p=0.028$, (b) $p=0.099$, (c) $p=0.827$, and (d) $p=0.254$

\# Weight $(\%)=$ relative weight $($ random)

OR: $1.02 ; 95 \%$ CI: $\left.0.94-1.11 ; p=0.6027 ; I^{2}: 96.08 \%\right)$ and hypertension $(r: 0.001$; OR: 1.00 ; 95\% CI: 0.94-1.06; $p=$ $\left.0.9685 ; I^{2}: 95.99 \%\right)$ had not been associated with increased odds of mortality or needs for IMV (Table 3 ).

Figures 1 and 2 show a forest plot of age-adjusted factors contributing poor outcomes amongst COVID-19 patients. Sensitivity analysis showed that the removal of any single study did not change the significance of the results. Unadjusted relationships are mentioned in the eSupplemental file (3).
eSupplemental file (4) shows age-adjusted meta-regression suggests incremental association between mortality (logevent) and pooled prevalence of male, ICU admission, IMV utilization, cerebrovascular disease, chronic liver disease, acute respiratory distress syndrome, septic shock, and cardiac complications. eSupplemental file (5) shows age-adjusted meta-regression suggests incremental association between IMV utilization (log-event) and pooled prevalence of male, cerebrovascular disease, chronic liver disease, cardiovascular disease, and acute respiratory distress syndrome. 
Table 2 Demographics, clinical features, and outcomes of patients with COVID-19

\begin{tabular}{|c|c|c|c|c|}
\hline Variable & $\begin{array}{l}\text { Number of } \\
\text { patients } \\
\text { affected }\end{array}$ & $\begin{array}{l}\text { Total } \\
\text { number } \\
\text { of patients }\end{array}$ & $\begin{array}{l}\text { Pooled } \\
\text { percentage } \\
\%(95 \% \mathrm{CI})^{*}\end{array}$ & $\begin{array}{l}\text { Heterogeneity } \\
\left(I^{2}\right) \%\end{array}$ \\
\hline \multicolumn{5}{|l|}{ Patient demographics } \\
\hline Age in years (median, range) & $52.5(41-70)$ & 12,247 & - & - \\
\hline Female & 5042 & 12,247 & $42.6(40.4-44.8)$ & 66.6 \\
\hline Males & 7198 & 12,247 & $57.3(55.1-59.4)$ & 66.4 \\
\hline \multicolumn{5}{|l|}{ Clinical features } \\
\hline Headache & 306 & 2738 & $10.7(7.9-14.3)$ & 79.1 \\
\hline Fever & 5172 & 9563 & $85.6(73.6-92.7)$ & 98.8 \\
\hline Cough & 2464 & 3863 & $64.7(57.4-71.4)$ & 93.7 \\
\hline Diarrhea & 400 & 3428 & $9.4(6.2-14.1)$ & 92.2 \\
\hline Dyspnea & 1259 & 3629 & $32(23.9-41.3)$ & 95.8 \\
\hline Hemoptysis & 29 & 1804 & $2.1(1.1-3.9)$ & 56.8 \\
\hline Myalgia/fatigue & 1848 & 3813 & $43.3(35.8-51.2)^{\mathrm{a}}$ & 94.5 \\
\hline Nausea/vomiting & 265 & 3258 & $7(4.4-10.8)$ & 90.6 \\
\hline Sore throat & 192 & 1344 & $17.3(9.1-30.3)$ & 85.9 \\
\hline Nasal congestion/rhinorrhea & 50 & 1082 & $7.5(3.1-17.4)$ & 88.1 \\
\hline Sputum production & 968 & 2846 & $33.4(29.1-38.1)$ & 79.4 \\
\hline \multicolumn{5}{|l|}{ Comorbidities } \\
\hline Smoker & 3003 & 8410 & $8.9(4.2-17.9)$ & 98.8 \\
\hline Diabetes & 2897 & 11,680 & $15.4(12-19.4)$ & 95.8 \\
\hline Hypertension & 4858 & 11,626 & $28.2(22.1-35.1)$ & 97.8 \\
\hline Malignancy & 578 & 11,486 & $4(3.1-5.2)$ & 76.6 \\
\hline Pulmonary disease & 1371 & 11,402 & $5.5(3.8-7.7)$ & 94.1 \\
\hline Chronic liver disease & 116 & 8830 & $3(1.4-6.1)$ & 92.6 \\
\hline Cerebrovascular disease & 244 & 4987 & $4.4(2.9-6.5)$ & 83.6 \\
\hline Cardiovascular disease & 2044 & 11,664 & $12.2(8.9-16.6)$ & 96.8 \\
\hline \multicolumn{5}{|l|}{ Complications } \\
\hline Pneumonia & 1518 & 2113 & $68.1(38.8-87.8)^{\mathrm{b}}$ & 98.3 \\
\hline $\begin{array}{l}\text { Acute respiratory distress } \\
\text { syndrome }\end{array}$ & 470 & 2518 & $29.9(18.5-44.7)^{\mathrm{c}}$ & 96.6 \\
\hline Septic shock & 68 & 1920 & $3.6(0.9-13.8)$ & 96.1 \\
\hline Secondary infection & 218 & 1187 & $13.8(5.8-29.3)$ & 96.1 \\
\hline Cardiac complications & 357 & 1246 & $22.3(12.8-36.1)$ & 95.1 \\
\hline Others & 268 & 2180 & $21.2(7.4-47.6)$ & 97.9 \\
\hline \multicolumn{5}{|l|}{ Clinical outcomes } \\
\hline Discharged/recovery & 3906 & 11,083 & $36.6(28.9-44.9)^{\mathrm{d}}$ & 97.6 \\
\hline ICU & 2038 & 10,230 & $18.8(14.7-23.8)$ & 92.5 \\
\hline Mechanical ventilation & 1789 & 8804 & $23.3(17.1-30.9)$ & 95.6 \\
\hline Mortality & 1267 & 11,252 & $13(9.3-18)$ & 95.6 \\
\hline
\end{tabular}

For the accuracy of the epidemiological characteristics, we have considered all the studies $(n=29)$ mentioning COVID-19 epidemiology with or without outcomes

*Statistically significant at $p=<0.001$ except (a) $p=0.096$, (b) $p=0.009$, (c) $p=0.009$, and (d) $p=0.002$

\section{Heterogeneity $\left(I^{2}\right)$ Statistics}

The heterogeneity analysis of the age-adjusted mortality and IMV showed 67-96\% and 77-96\% dispersion observed between studies, respectively. Additionally, overall studies had moderate risk of bias (eSupplemental file (6)).

\section{Discussion}

In our meta-regression analysis of 29 observational studies with 12,258 confirmed cases of COVID-19 patients, the pooled prevalence of IMV was $23.3 \%$, and mortality was $13 \%$. Male $(57.3 \%)$ and those with pre-existing hypertension 


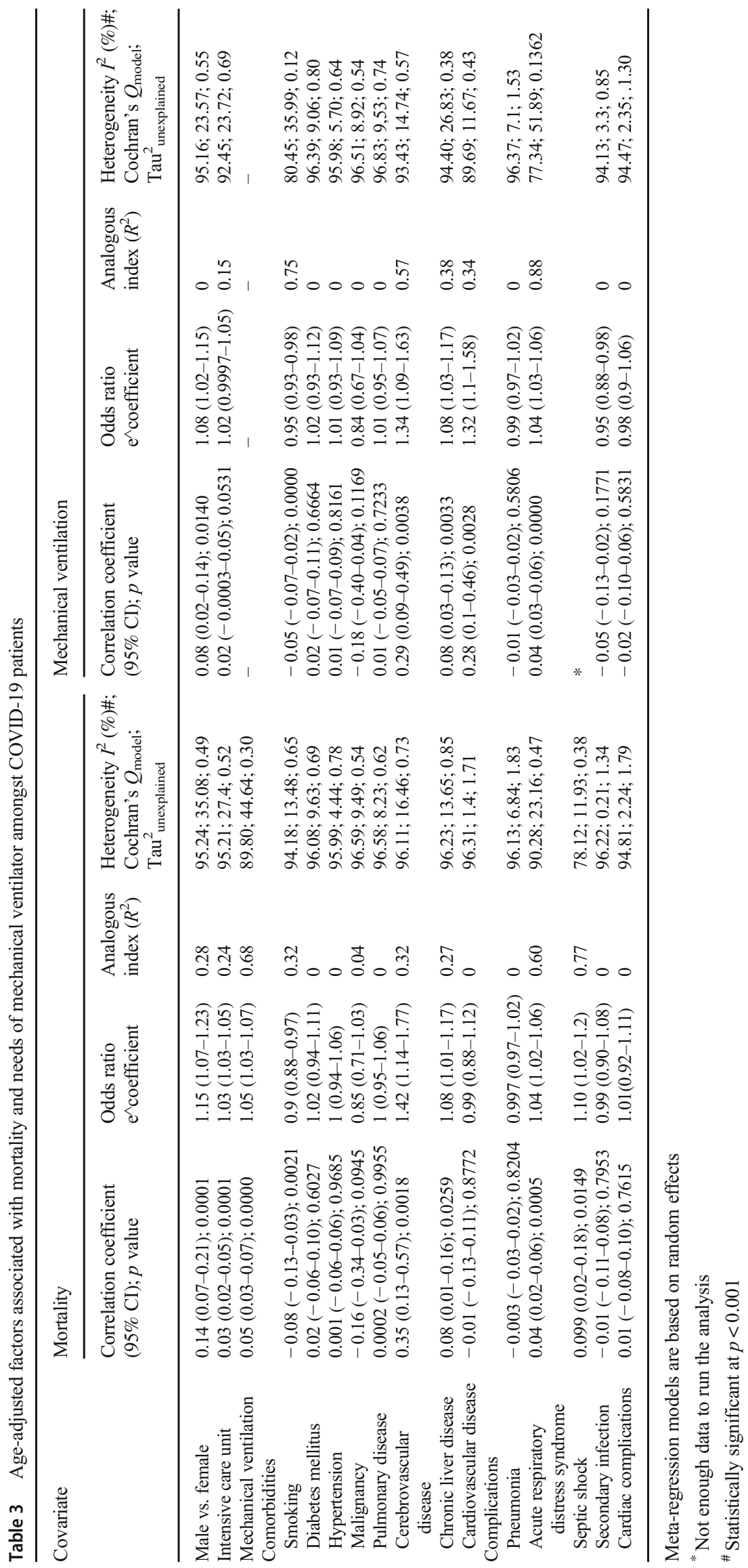


Fig. 1 Forest plot of age-adjusted factors contributing to mortality amongst COVID-19 patients
Factors contributing mortality amongst COVID-19 patients

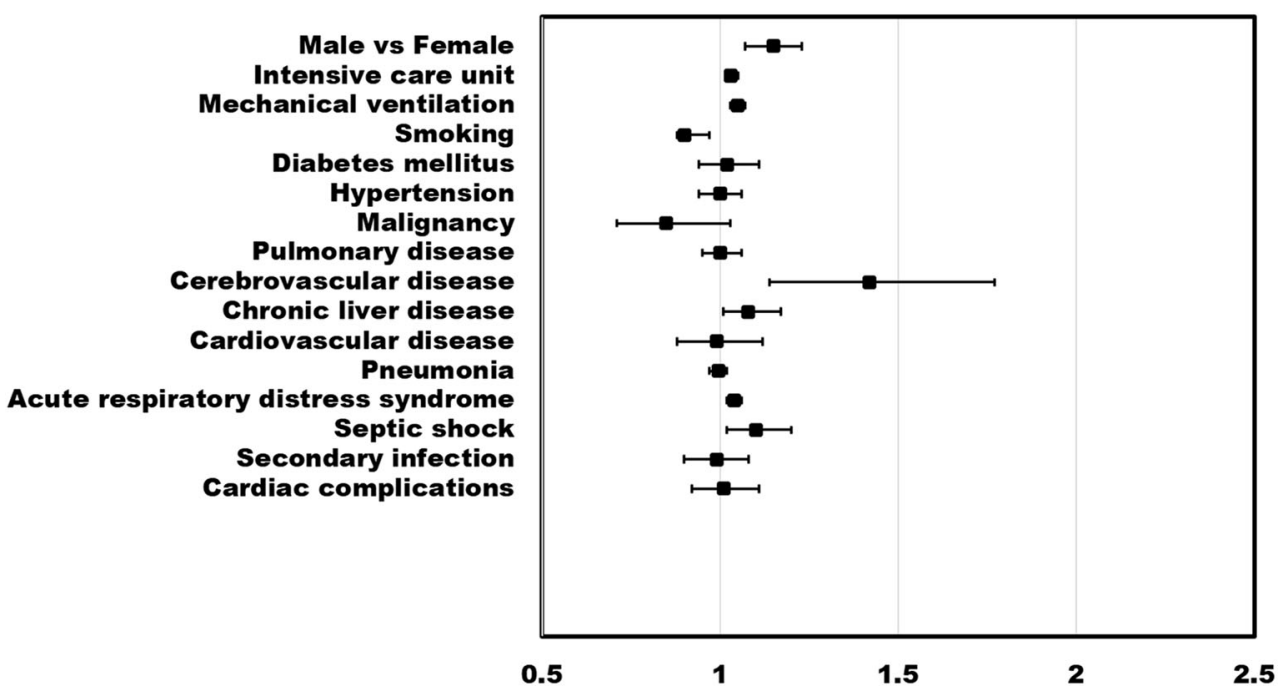

(28.2\%), diabetes $(15.4 \%)$, cardiovascular disease $(12.2 \%)$, and cerebrovascular diseases $(4.4 \%)$ had the highest prevalence in our study cohort. Our results are consistent with other studies from China and outside China [3, 6, 11, 15-18]. Regardless of the variations in the sample size and the geographical locations, cardiovascular disease and hypertension remain the most common comorbidity PM [15, 19-22]. The mortality rate for SARS-CoV was more than $10 \%$ and for MERS-CoV was more than $35 \%$, and both are highly pathogenic organisms $[23,24]$. The decreased vulnerability of females to viral infections may be assigned to X chromosome and sex hormone protectiveness, both of which play an important role in innate and adaptive immunity [25].

Furthermore, studies have reported that the majority of the COVID-19 patients had coexisting comorbidities, mainly cardiovascular and cerebrovascular diseases [17] and diabetes, similar to MERS-CoV [26] or any type of severe infectious disease that require hospital or ICU admission [27]. In our
Fig. 2 Forest plot of age-adjusted factors contributing to mechanical ventilation amongst COVID-19 patients

\section{Factors contributing Mechanical ventilation amongst COVID-19 patients}

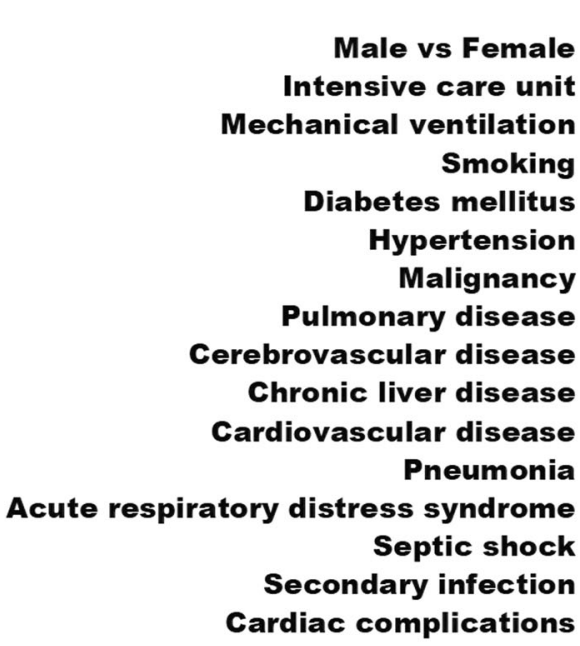

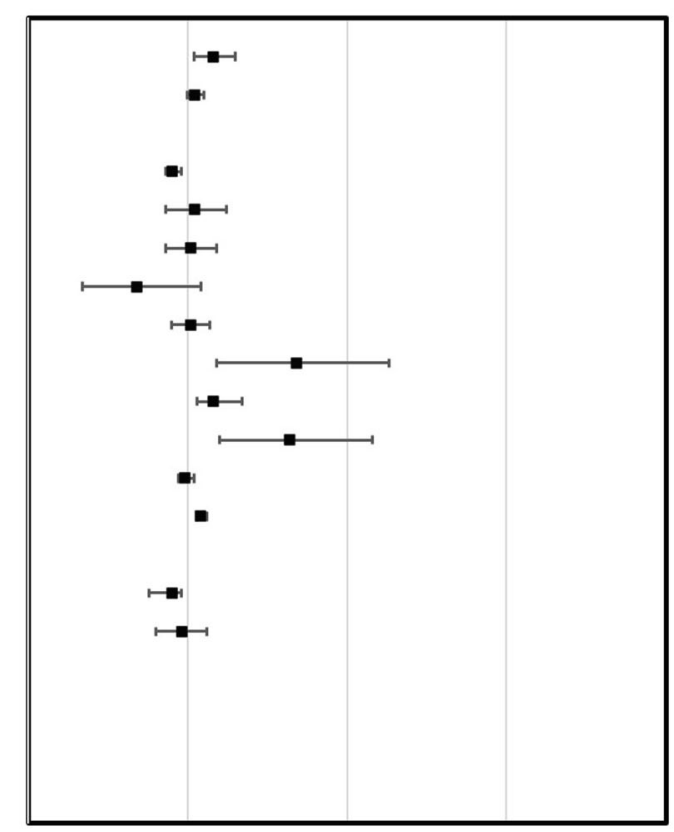

$\begin{array}{lllll}0.5 & 1 & 1.5 & 2 & 2.5\end{array}$ 
study, comorbidities like pre-existing cerebrovascular disease, cardiovascular disease, and chronic liver disease were significantly associated with increased odds of mortality and IMV utilization in COVID-19 patients. The outcomes in many studies are similar to ours $[16,28]$. It is well known that some comorbidities frequently coexist, and such patients are more likely to have poor well-being. A study by Guan et al. has found significantly increased risk of poor outcomes in COVID-19 patients with at least one comorbidity, or even more compared with patients with no comorbidity [29]. They also reported that severe cases were more likely to have hypertension, cardiovascular diseases, cerebrovascular diseases, and diabetes compared with non-severe cases, suggesting that both the category and number of comorbidities should be taken into account when predicting COVID-19 patients' prognosis. There is an assumption that immune dysregulation and prolonged inflammation might be the key drivers of the poor clinical outcomes in COVID-19 but await verification in more mechanistic studies [29].

However, we found no association of hypertension and diabetes with mortality and IMV. To support our findings, a study predicting factors associated with mortality in COVID19 pneumonia reported that mortality was not associated with malignancy or diabetes [10]. Until now, it is not evident whether the severity or level of control of pre-existing health conditions has affected the risk for severe disease in COVID19 patients. Additionally, many of these comorbidities have high prevalence in the USA. According to the AHA 2020 report [30], the prevalence of cardiovascular disease (excluding hypertension) was $10.6 \%$. Considering the findings of our study, both highly prevalent comorbidities in COVID-19 patients in the USA and potential risk for more severe COVID19 disease in patients with these comorbidities highlight the importance of COVID-19 prevention in people with underlying health conditions. Therefore, CDC continues to develop and update resources for persons with underlying health conditions to reduce the risk of acquiring COVID-19 [31].

Interestingly, there has not been published literature on the association of COVID-19 complications with poor outcomes. To our knowledge, this is the first study to report that COVID19 patients with complications of ARDS have higher odds of mortality and IMV compared with those without ARDS. Hence, our study findings have added to the existing literature of common coexisting comorbidities and complications in patients with COVID-19 and its associated outcomes based on the large sample size and representing global population.

\section{Strength and Limitations}

To our knowledge, this is the first large population study that shows association between risk factors and outcomes, using meta-regression of 12,258 RT-PCR confirmed COVID-19 patients. Our findings may provide early insights into designing models for early identification of high-risk patients and prioritizing their treatment based on disease severity, which will help in prudent use of limited healthcare resources during this pandemic. A limitation of this study is missing details on severity of these risk factors. In addition, we have analyzed the group data of COVID-19 hospitalized patients, and individual patient meta-analysis would probably be able to better tease out relationships between multiple factors and reduce the risk of ecological fallacy while attempting to make inferences about individuals using study-level information. Also, since the primary studies are from very different healthcare systems, there may be uncaptured differences in ancillary care, criteria for IMV, ICU care, and etc. Due to non-identical effects being estimated in studies analyzed in our meta-regression, our study has high heterogeneity which we tried to justify using random-effects model and sensitivity analysis.

\section{Conclusion}

Our study suggests that COVID-19 patients with coexisting comorbidities such as cardiovascular disease, cerebrovascular disease, and chronic liver disease had poor outcomes of death and IMV compared with those without it. Hence, our study results might be helpful for clinicians in proper triage of patients by watchfully talking about the medical history, as this will help in early identification of high-risk patients who would be more likely to develop serious adverse outcomes of COVID-19 which in turn will be helpful in appropriate allocation of healthcare resources. However, diabetes and hypertension had higher prevalence in the study cohort but no association with mortality and IMV. Future studies should focus specifically on these comorbidities and their associated outcomes.

Authors' Contributions Conceptualization: UP; methodology: UP, PM; acquisition of data: UP, PM; formal analysis and investigation: UP, PM, MSU, TJS; writing — original draft preparation: UP, PM, MSU, DM, AS, FAM, NK; writing - review, critical feedback, and editing: JA, AP, HS; funding acquisition: none; resources: HS; supervision: HS.

Data Availability The data is collected from the studies published online, publicly available, and specific details related to data and/or analysis will be made available upon request.

\section{Compliance with Ethical Standards}

Conflict of Interest The authors declare that they have no conflict of interest.

Ethical Approval Though this article does not contain any studies with direct involvement of human participants or animals performed by any of the authors, all procedures performed in studies involving human participants were in accordance with the ethical standards of the institutional and/or national research committee and with the 1964 Helsinki declaration and its later amendments or comparable ethical standards. 
Informed Consent The data used in this study is deidentified and collected from the studies published online; thus, informed consent or IRB approval was not needed for this study.

\section{References}

1. Holshue ML, DeBolt C, Lindquist S, Lofy KH, Wiesman J, Bruce $\mathrm{H}$, et al. First case of 2019 novel coronavirus in the United States. N Engl J Med. 2020;382(10):929-36. https://doi.org/10.1056/ NEJMoa2001191.

2. Coronavirus disease 2019 (COVID-19)-cases in the U.S. Centers for Disease Control and Prevention. 2020. https://www.cdc.gov/ coronavirus/2019-ncov/cases-updates/cases-in-us.html. Accessed June 32020

3. Goyal P, Choi JJ, Pinheiro LC, Schenck EJ, Chen R, Jabri A, et al. Clinical characteristics of Covid-19 in New York City. N Engl J Med. 2020;382:2372-4. https://doi.org/10.1056/NEJMc2010419.

4. Sanyaolu A, Okorie C, Marinkovic A, Patidar R, Younis K, Desai $\mathrm{P}$, et al. Comorbidity and its impact on patients with COVID-19. Sn Compr Clin Med. 2020;2:1-8. https://doi.org/10.1007/s42399-02000363-4.

5. Ruan Q, Yang K, Wang W, Jiang L, Song J. Clinical predictors of mortality due to COVID-19 based on an analysis of data of 150 patients from Wuhan, China. Intensive Care Med. 2020;46(5):8468. https://doi.org/10.1007/s00134-020-05991-x.

6. Richardson S, Hirsch JS, Narasimhan M, Crawford JM, McGinn T, Davidson KW, et al. Presenting characteristics, comorbidities, and outcomes among 5700 patients hospitalized with COVID-19 in the New York City area. JAMA. 2020;323(20):2052-9. https://doi.org/ 10.1001/jama.2020.6775.

7. Yang X, Yu Y, Xu J, Shu H, Ja X, Liu H, et al. Clinical course and outcomes of critically ill patients with SARS-CoV-2 pneumonia in Wuhan, China: a single-centered, retrospective, observational study. Lancet Respir Med. 2020;8(5):475-81. https://doi.org/10. 1016/S2213-2600(20)30079-5.

8. Diabetes Statistics. Diabetes Research Institute. https://www. diabetesresearch.org/diabetes-statistics.

9. Fryar CD, Ostchega Y, Hales CM, Zhang G, Kruszon-Moran D. Hypertension prevalence and control among adults: United States, 2015-2016. NCHS Data Brief. 2017;(289):1-8.

10. Du R-H, Liang L-R, Yang C-Q, Wang W, Cao T-Z, Li M, et al. Predictors of mortality for patients with COVID-19 pneumonia caused by SARS-CoV-2: a prospective cohort study. Eur Respir J. 2020:2000524. https://doi.org/10.1183/13993003.00524-2020.

11. Zhou F, Yu T, Du R, Fan G, Liu Y, Liu Z, et al. Clinical course and risk factors for mortality of adult inpatients with COVID-19 in Wuhan, China: a retrospective cohort study. Lancet. 2020;395(10229):1054-62. https://doi.org/10.1016/S01406736(20)30566-3.

12. Roy S, Mazumder T, Banik S. The association of cardiovascular diseases and diabetes mellitus with COVID-19 (SARS-CoV-2) and their possible mechanisms. Sn Compr Clin Med. 2020;2:1-6. https://doi.org/10.1007/s42399-020-00376-z.

13. Stroup DF, Berlin JA, Morton SC, Olkin I, Williamson GD, Rennie D, et al. Meta-analysis of observational studies in epidemiology: a proposal for reporting. Meta-analysis Of Observational Studies in Epidemiology (MOOSE) group. JAMA. 2000;283(15):2008-12. https://doi.org/10.1001/jama.283.15.2008.

14. Wells GA, Shea B, O'Connell D, Peterson J, Welch V, Losos M, et al. The Newcastle-Ottawa Scale (NOS) for assessing the quality of nonrandomised studies in meta-analyses. Professor GA Wells, Department of Epidemiology and Commuunity Medicine, University of Ottawa, Ontario K1J 8M5, Canada. 2020. http:// www.ohri.ca/programs/clinical_epidemiology/oxford.asp.
15. Chen N, Zhou M, Dong X, Qu J, Gong F, Han Y, et al. Epidemiological and clinical characteristics of 99 cases of 2019 novel coronavirus pneumonia in Wuhan, China: a descriptive study. Lancet. 2020;395(10223):507-13. https://doi.org/10.1016/ s0140-6736(20)30211-7.

16. Preliminary estimates of the prevalence of selected underlying health conditions among patients with coronavirus disease 2019United States, February 12-March 28, 2020. MMWR Morb Mortal Wkly Rep. 2020;69(13):382-6. https://doi.org/10.15585/mmwr. mm6913e2.

17. Patel U, Malik P, Shah D, Patel A, Dhamoon M, Jani V. Preexisting cerebrovascular disease and poor outcomes of COVID19 hospitalized patients: a meta-analysis. J Neurol. 2020. https:// doi.org/10.1007/s00415-020-10141-w.

18. Kaur N, Gupta I, Singh H, Karia R, Ashraf A, Habib A, et al. Epidemiological and clinical characteristics of 6635 COVID-19 patients: a pooled analysis. SN Compr Clin Med. 2020;2(8): 1048-52. https://doi.org/10.1007/s42399-020-00393-y.

19. Huang C, Wang Y, Li X, Ren L, Zhao J, Hu Y, et al. Clinical features of patients infected with 2019 novel coronavirus in Wuhan, China. Lancet. 2020;395(10223):497-506. https://doi. org/10.1016/s0140-6736(20)30183-5.

20. Wang D, Hu B, Hu C, Zhu F, Liu X, Zhang J, et al. Clinical characteristics of 138 hospitalized patients with 2019 novel coronavirus-infected pneumonia in Wuhan, China. JAMA. 2020;323(11):1061-9. https://doi.org/10.1001/jama.2020.1585.

21. Liu K, Fang Y-Y, Deng Y, Liu W, Wang M-F, Ma J-P, et al. Clinical characteristics of novel coronavirus cases in tertiary hospitals in Hubei Province. Chin Med J. 2020;133(9):1025-31. https:// doi.org/10.1097/cm9.0000000000000744.

22. Xu XW, Wu XX, Jiang XG, Xu KJ, Ying LJ, Ma CL, et al. Clinical findings in a group of patients infected with the 2019 novel coronavirus (SARS-Cov-2) outside of Wuhan, China: retrospective case series. BMJ. 2020;368:m606. https://doi.org/10.1136/bmj.m606.

23. Yin Y, Wunderink RG. MERS, SARS and other coronaviruses as causes of pneumonia. Respirology. 2018;23(2):130-7. https://doi. org/10.1111/resp.13196.

24. Song Z, Xu Y, Bao L, Zhang L, Yu P, Qu Y, et al. From SARS to MERS, thrusting coronaviruses into the spotlight. Viruses. 2019;11(1). https://doi.org/10.3390/v11010059.

25. Jaillon S, Berthenet K, Garlanda C. Sexual dimorphism in innate immunity. Clin Rev Allergy Immunol. 2019;56(3):308-21. https:// doi.org/10.1007/s12016-017-8648-x.

26. Badawi A, Ryoo SG. Prevalence of comorbidities in the Middle East respiratory syndrome coronavirus (MERS-CoV): a systematic review and meta-analysis. Int J Infect Dis. 2016;49:129-33. https:// doi.org/10.1016/j.ijid.2016.06.015.

27. Cecconi M, Evans L, Levy M, Rhodes A. Sepsis and septic shock. Lancet. 2018;392(10141):75-87. https://doi.org/10.1016/s01406736(18)30696-2.

28. Petrilli CM, Jones SA, Yang J, Rajagopalan H, O'Donnell L, Chernyak Y, et al. Factors associated with hospital admission and critical illness among 5279 people with coronavirus disease 2019 in New York City: prospective cohort study. BMJ. 2020;369:m1966. https://doi.org/10.1136/bmj.m1966.

29. Guan W-j, Liang W-h, Zhao Y, Liang H-r, Chen Z-s, Li Y-m, et al. Comorbidity and its impact on 1590 patients with COVID-19 in China: a nationwide analysis. Eur Respir J. 2020;55(5):2000547. https://doi.org/10.1183/13993003.00547-2020.

30. Virani SS, Alonso A, Benjamin EJ, Bittencourt MS, Callaway CW, Carson AP, et al. Heart disease and stroke statistics-2020 update: a report from the American Heart Association. Circulation. 2020;141(9):e139-596. https://doi.org/10.1161/cir. 0000000000000757.

31. People who are at higher risk for severe illness. Centers for Disease Control and Prevention. 2020. https://www.cdc.gov/coronavirus/ 
2019-ncov/need-extra-precautions/people-at-higher-risk.html? CDC_AA_refVal=https\%3A\%2F\%2Fwww.cdc.gov\% 2Fcoronavirus\%2F2019-ncov\%2Fspecific-groups\%2Fhigh-riskcomplications.html. Accessed June 22020.
Publisher's Note Springer Nature remains neutral with regard to jurisdictional claims in published maps and institutional affiliations.

\section{Affiliations}

Urvish Patel $^{1}$ • Preeti Malik ${ }^{1}$ (D) $\cdot$ Muhammad Shariq Usman ${ }^{2} \cdot$ Deep Mehta $^{3} \cdot$ Ashish Sharma $^{4} \cdot$ Faizan Ahmad Malik $^{5}$. Nashmia Khan ${ }^{1} \cdot$ Tariq Jamal Siddiqi $^{2} \cdot$ Jawad Ahmed $^{2} \cdot$ Achint Patel $^{1} \cdot$ Henry Sacks $^{6}$

1 Department of Public Health, Icahn School of Medicine at Mount Sinai, 1 Gustave L. Levy Pl, New York, NY 10029, USA

2 Department of Internal Medicine, Dow University of Health Sciences, Karachi, Pakistan

3 Clinical Research Program, Icahn School of Medicine at Mount Sinai, New York, NY, USA
4 Department of Internal Medicine, Yuma Regional Medicine, Yuma, AZ, USA

5 Department of Internal Medicine, Texas Tech University Health Sciences Center at Permian Basin, Odessa, TX, USA

6 Department of Environmental Medicine and Public Health, Icahn School of Medicine at Mount Sinai, New York, NY, USA 